\title{
Arithmetic Operations on Short Finite Sequences
}

\author{
Rafał Ziobro \\ Department of Carbohydrate Technology \\ University of Agriculture \\ Krakow, Poland
}

\begin{abstract}
Summary. In contrast to other proving systems Mizar Mathematical Library, considered as one of the largest formal mathematical libraries 4], is maintained as a single base of theorems, which allows the users to benefit from earlier formalized items [3], 2]. This eventually leads to a development of certain branches of articles using common notation and ideas. Such formalism for finite sequences has been developed since 1989 [1] and further developed despite of the controversy over indexing which excludes zero 6, also for some advanced and new mathematics [5].

The article aims to add some new machinery for dealing with finite sequences, especially those of short length.
\end{abstract}

MSC: 11B99 03B35 68T99

Keywords: finite sequences; functions; relations

MML identifier: FINSEQ_9, version: 8.1.08 5.53.1335

\section{Preliminaries}

One can verify that every binary relation which is empty is also positive yielding and every binary relation which is empty is also negative yielding and every binary relation which is natural-valued is also $\mathbb{N}$-valued.

Let $f$ be a complex-valued function and $k$ be an object. Note that $(0 \cdot f)(k)$ reduces to 0 .

Let us observe that $1 \cdot f$ reduces to $f$ and $(-1) \cdot(-f)$ reduces to $f$. One can verify that $0 \cdot f$ is empty yielding and $f-f$ is empty yielding. 
Let $D$ be a set. Observe that there exists a $D$-valued finite sequence which is empty yielding and every finite sequence which is empty yielding is also $\mathbb{N}$-valued and there exists an empty yielding finite sequence which is non empty.

Let $n$ be a natural number. One can verify that there exists an empty yielding, $\mathbb{N}$-valued finite sequence which is $n$-element and $\min (n, 0)$ is zero.

One can verify that $\max (n, 0)$ reduces to $n$.

Let $a$ be a non zero natural number. One can verify that $\min (a, 1)$ reduces to 1 and $\max (a, 1)$ reduces to $a$.

Let $a$ be a non trivial natural number. One can verify that $\min (a, 2)$ reduces to 2 and $\max (a, 2)$ reduces to $a$.

Let $a$ be a positive real number and $b$ be a positive natural number. One can verify that $b \mapsto a$ is positive and every binary relation which is empty yielding is also function-like and every function which is empty yielding is also natural-valued and every real-valued function which is empty yielding is also non-positive yielding.

Every real-valued function which is empty yielding is also non-negative yielding and every non empty, real-valued function which is empty yielding is also non positive yielding and every non empty, real-valued function which is empty yielding is also non negative yielding and every non empty, real-valued function which is positive yielding is also non non-positive yielding and every non empty, real-valued function which is negative yielding is also non non-negative yielding.

Let $f$ be an empty yielding function and $c$ be a complex number. Note that $c \cdot f$ is empty yielding.

Let $g$ be a complex-valued function. Note that $f \cdot g$ is empty yielding.

\section{The Length of Finite Sequences}

Let $f$ be a complex-valued finite sequence and $x$ be a complex number. Note that $f+x$ is $(\operatorname{len} f)$-element and $f-x$ is $(\operatorname{len} f)$-element and $|f|$ is (len $f)$-element and $-f$ is (len $f$ )-element and $f^{-1}$ is (len $f$ )-element.

Let $n, m$ be natural numbers, $f$ be an $n$-element, complex-valued finite sequence, and $g$ be an $m$-element, complex-valued finite sequence. One can verify that $f+g$ is $(\min (n, m))$-element and $f \cdot g$ is $(\min (n, m))$-element and $f-g$ is $(\min (n, m))$-element and $f / g$ is $(\min (n, m))$-element.

Let $g$ be an $(n+m)$-element, empty yielding, complex-valued finite sequence. Observe that $f+g$ reduces to $f$.

Let $n$ be a natural number and $g$ be an $n$-element, empty yielding, complexvalued finite sequence. One can verify that $f+g$ reduces to $f$.

Let $X$ be a non empty set. Observe that there exists an $X$-defined, empty yielding function which is total. 
Let $f$ be a total, $X$-defined, complex-valued function and $g$ be a total, $X$-defined, empty yielding function. Let us observe that $f+g$ reduces to $f$.

Let $f$ be a binary relation. Let us observe that there exists a binary relation which is $(\operatorname{dom} f)$-defined and $f$ null $f$ is $(\operatorname{dom} f)$-defined and there exists a $(\operatorname{dom} f)$-defined binary relation which is total.

Let $f$ be a complex-valued function. Observe that there exists a $(\operatorname{dom} f)$ defined, empty yielding function which is total and $-f$ is $(\operatorname{dom} f)$-defined and $-f$ is total and $f^{-1}$ is $(\operatorname{dom} f)$-defined and $f^{-1}$ is total and $|f|$ is $(\operatorname{dom} f)$-defined and $|f|$ is total.

Let $c$ be a complex number. Let us note that $c+f$ is $(\operatorname{dom} f)$-defined and $c+f$ is total and $f-c$ is $(\operatorname{dom} f)$-defined and $f-c$ is total and $c \cdot f$ is $(\operatorname{dom} f)$-defined and $c \cdot f$ is total.

Let $f$ be a finite sequence. Let us observe that every finite sequence which is (len $f$ )-element is also $(\operatorname{dom} f)$-defined.

Let $n$ be a natural number. Let us observe that every finite sequence which is $n$-element is also ( $\operatorname{Seg} n$ )-defined and every finite sequence which is total and $(\operatorname{Seg} n)$-defined is also $n$-element.

Now we state the proposition:

(1) Let us consider a complex-valued finite sequence $f$. Then $0 \cdot f=\operatorname{len} f \mapsto$ 0 .

Let $f$ be a complex-valued finite sequence. Note that $f+$ len $f \mapsto 0$ reduces to $f$.

Let $n$ be a natural number, $D$ be a non empty set, and $X$ be a non empty subset of $D$. One can verify that there exists an $X$-valued finite sequence which is $n$-element and there exists a finite sequence of elements of $X$ which is $n$-element.

\section{On Positive and Negative Yielding Functions}

Let $f$ be a real-valued function. Let us note that $f+|f|$ is non-negative yielding and $|f|-f$ is non-negative yielding.

Let $f$ be a non-negative yielding, real-valued function and $x$ be an object. Observe that $f(x)$ is non negative.

Let $f$ be a non-positive yielding, real-valued function. Let us observe that $f(x)$ is non positive.

Let $f$ be a non-negative yielding, real-valued function and $r$ be a non negative real number. One can verify that $r \cdot f$ is non-negative yielding and $(-r) \cdot f$ is non-positive yielding and $-f$ is non-positive yielding.

Let $f$ be a non-positive yielding, real-valued function and $r$ be a non negative real number. Let us observe that $r \cdot f$ is non-positive yielding and $(-r) \cdot f$ is non- 
negative yielding and $-f$ is non-negative yielding and every $\mathbb{Z}$-valued function which is non-negative yielding is also natural-valued.

Let $f$ be a $\mathbb{Z}$-valued function. Let us observe that $\frac{1}{2} \cdot(f+|f|)$ is natural-valued and $\frac{1}{2} \cdot(|f|-f)$ is natural-valued.

Let us consider a binary relation $f$. Now we state the propositions:

(2) $\operatorname{rng} f$ is natural-membered if and only if $f$ is natural-valued. Proof: If $\operatorname{rng} f$ is natural-membered, then $f$ is natural-valued.

(3) $\quad f$ is $\mathbb{N}$-valued if and only if $\operatorname{rng} f$ is natural-membered. The theorem is a consequence of $(2)$.

(4) $\operatorname{rng} f$ is integer-membered if and only if $f$ is $\mathbb{Z}$-valued. Proof: If $\operatorname{rng} f$ is integer-membered, then $f$ is $\mathbb{Z}$-valued.

(5) $\quad \operatorname{rng} f$ is rational-membered if and only if $f$ is $\mathbb{Q}$-valued. Proof: If $\operatorname{rng} f$ is rational-membered, then $f$ is $\mathbb{Q}$-valued.

(6) $\operatorname{rng} f$ is real-membered if and only if $f$ is real-valued. PROOF: If $\operatorname{rng} f$ is real-membered, then $f$ is real-valued.

(7) $\quad f$ is $\mathbb{R}$-valued if and only if $\operatorname{rng} f$ is real-membered. The theorem is a consequence of (6).

(8) $\operatorname{rng} f$ is complex-membered if and only if $f$ is complex-valued. Proof: If rng $f$ is complex-membered, then $f$ is complex-valued.

(9) $f$ is $\mathbb{C}$-valued if and only if $\operatorname{rng} f$ is complex-membered. The theorem is a consequence of (8).

(10) $\operatorname{dom} f$ is natural-membered if and only if $f$ is $\mathbb{N}$-defined.

Proof: If $\operatorname{dom} f$ is natural-membered, then $f$ is $\mathbb{N}$-defined.

Let $f$ be a $\mathbb{Z}$-defined binary relation. Observe that $\operatorname{dom} f$ is integer-membered. Now we state the proposition:

(11) Let us consider a binary relation $f$. Then $\operatorname{dom} f$ is integer-membered if and only if $f$ is $\mathbb{Z}$-defined.

Proof: If $\operatorname{dom} f$ is integer-membered, then $f$ is $\mathbb{Z}$-defined.

Let $f$ be a $\mathbb{Q}$-defined binary relation. Let us note that $\operatorname{dom} f$ is rationalmembered.

Now we state the proposition:

(12) Let us consider a binary relation $f$. Then $\operatorname{dom} f$ is rational-membered if and only if $f$ is $\mathbb{Q}$-defined.

Proof: If $\operatorname{dom} f$ is rational-membered, then $f$ is $\mathbb{Q}$-defined.

Let $f$ be a $\mathbb{R}$-defined binary relation. Note that $\operatorname{dom} f$ is real-membered.

Now we state the proposition:

(13) Let us consider a binary relation $f$. Then $\operatorname{dom} f$ is real-membered if and only if $f$ is $\mathbb{R}$-defined. 
Proof: If $\operatorname{dom} f$ is real-membered, then $f$ is $\mathbb{R}$-defined.

Let $f$ be a $\mathbb{C}$-defined binary relation. One can check that $\operatorname{dom} f$ is complexmembered.

Now we state the propositions:

(14) Let us consider a binary relation $f$. Then $\operatorname{dom} f$ is complex-membered if and only if $f$ is $\mathbb{C}$-defined.

PRoOF: If $\operatorname{dom} f$ is complex-membered, then $f$ is $\mathbb{C}$-defined.

(15) Let us consider a set $D$, and a function $f$. Then $f$ is $D$-valued if and only if $f$ is a function from $\operatorname{dom} f$ into $D$.

Proof: If $f$ is $D$-valued, then $f$ is a function from dom $f$ into $D$.

(16) Let us consider a set $C$. Then every total, $C$-defined function is a function from $C$ into $\operatorname{rng} f$.

(17) Let us consider sets $C, D$, and a total, $C$-defined function $f$. Then $f$ is a function from $C$ into $D$ if and only if $f$ is $D$-valued. The theorem is a consequence of (16) and (15).

(18) Every real-valued function is a function from $\operatorname{dom} f$ into $\mathbb{R}$.

(19) Let us consider a complex-valued finite sequence $f$. Then

(i) $f-f=0 \cdot f$, and

(ii) $f-f=\operatorname{len} f \mapsto 0$.

The theorem is a consequence of (1).

(20) Let us consider a complex number $a$, a finite sequence $f$, and a natural number $k$. If $k \in \operatorname{dom} f$, then $(\operatorname{len} f \mapsto a)(k)=a$.

Let $a$ be a real number, $k$ be a non zero natural number, $l$ be a natural number, and $f$ be a $(k+l)$-element finite sequence. One can verify that (len $f \mapsto$ $a)(k)$ reduces to $a$.

Let $f$ be a complex-valued function. The functors: $\operatorname{delneg} f$, delpos $f$, and delall $f$ yielding complex-valued functions are defined by terms

(Def. 1) $\frac{1}{2} \cdot(f+|f|)$,

(Def. 2) $\frac{1}{2} \cdot(|f|-f)$,

(Def. 3) $0 \cdot f$,

respectively. Now we state the propositions:

(21) Let us consider a complex-valued function $f$. Then

(i) $\operatorname{dom} f=\operatorname{dom}(\operatorname{delpos} f)$, and

(ii) $\operatorname{dom} f=\operatorname{dom}(\operatorname{delneg} f)$, and

(iii) $\operatorname{dom} f=\operatorname{dom}(\operatorname{delall} f)$. 
(22) Let us consider a complex-valued function $f$, and an object $x$. Then $f(x)=(\operatorname{delneg} f)(x)-(\operatorname{delpos} f)(x)$. The theorem is a consequence of (21).

(23) Let us consider a complex-valued function $f$. Then $f=\operatorname{delneg} f-$ delpos $f$. The theorem is a consequence of (21) and (22).

Let us consider a real-valued function $f$ and an object $x$. Now we state the propositions:

(i) $f(x)=(\operatorname{delneg} f)(x)$, or

(ii) $f(x)=-(\operatorname{delpos} f)(x)$.

The theorem is a consequence of (21).

(i) $(\operatorname{delneg} f)(x)=0$, or

(ii) $(\operatorname{delpos} f)(x)=0$.

The theorem is a consequence of (22) and (24).

Let $f$ be a real-valued function. One can verify that $\operatorname{delneg} f \cdot \operatorname{delpos} f$ is empty yielding.

Now we state the proposition:

(26) Let us consider a real-valued function $f$. Then delall $f=\operatorname{delneg} f$. delpos $f$. The theorem is a consequence of (21).

Let $f$ be a complex-valued function and $f_{1}$ be a total, (dom $f$ )-defined, empty yielding function. Let us observe that $f+f_{1}$ reduces to $f$ and $f-f_{1}$ reduces to $f$.

Let $f_{1}$ be a total, (dom $f$ )-defined, complex-valued function and $f_{2}$ be a total, (dom $f$ )-defined, empty yielding function. One can verify that $f_{1}+f_{2}$ reduces to $f_{1}$ and $f_{1}-f_{2}$ reduces to $f_{1}$.

Observe that $f-f$ is $(\operatorname{dom} f)$-defined and $f-f$ is total.

Now we state the proposition:

(27) Let us consider a complex-valued function $f$. Then $|f|=\operatorname{delneg} f+$ $\operatorname{delpos} f$.

Let $f$ be an empty finite sequence. Let us note that $\prod f$ is natural and $\prod f$ is non zero.

Let $f$ be a positive yielding, real-valued finite sequence. One can check that $\prod f$ is positive.

Let $f$ be a complex-valued finite sequence. Let us note that $\operatorname{delneg} f$ is (len $f$ )-element and delpos $f$ is (len $f$ )-element.

Now we state the proposition:

(28) Let us consider a complex-valued function $f$. Then $\operatorname{delneg} f=\operatorname{delpos}(-f)$.

Let $f$ be a non-negative yielding, real-valued function. Note that $|f|$ reduces to $f$ and delneg $f$ reduces to $f$. We identify delall $f$ with delpos $f$. We identify 
delpos $f$ with delall $f$. Let $f$ be a non-positive yielding, real-valued function. Observe that - delpos $f$ reduces to $f$. One can verify that delneg $f$ is empty yielding.

We identify delall $f$ with delneg $f$. We identify delneg $f$ with delall $f$. Now we state the proposition:

(29) Let us consider a finite sequence $f$ of elements of $\mathbb{Z}$. Then there exist finite sequences $f_{1}, f_{2}$ of elements of $\mathbb{N}$ such that $f=f_{1}-f_{2}$. The theorem is a consequence of (23).

Let $a$ be an integer and $n$ be a natural number. Note that $n \mapsto a$ is $\mathbb{Z}$-valued. Let $f$ be a non empty, empty yielding finite sequence. Observe that $\prod f$ is zero.

Now we state the propositions:

(30) Let us consider finite sequences $f_{1}, f_{2}$ of elements of $\mathbb{R}$. Suppose len $f_{1}=$ len $f_{2}$ and for every element $k$ of $\mathbb{N}$ such that $k \in \operatorname{dom} f_{1}$ holds $f_{1}(k) \geqslant$ $f_{2}(k)>0$. Then $\prod f_{1} \geqslant \prod f_{2}$.

Proof: For every element $k$ of $\mathbb{N}$ such that $k \in \operatorname{dom} f_{2}$ holds $f_{1}(k) \geqslant$ $f_{2}(k)>0$.

(31) Let us consider a real number $a$, and a finite sequence $f$ of elements of $\mathbb{R}$. Suppose for every element $k$ of $\mathbb{N}$ such that $k \in \operatorname{dom} f$ holds $0<f(k) \leqslant a$. Then $\prod f \leqslant \prod($ len $f \mapsto a)$. The theorem is a consequence of (20).

(32) Let us consider a non negative real number $a$, and a finite sequence $f$ of elements of $\mathbb{R}$. Suppose for every natural number $k$ such that $k \in \operatorname{dom} f$ holds $f(k) \geqslant a$. Then $\prod f \geqslant a^{\text {len } f}$. The theorem is a consequence of $(20)$.

(33) Let us consider non-negative yielding finite sequences $f_{1}, f_{2}$ of elements of $\mathbb{R}$. Suppose len $f_{1}=$ len $f_{2}$ and for every element $k$ of $\mathbb{N}$ such that $k \in \operatorname{dom} f_{2}$ holds $f_{1}(k) \geqslant f_{2}(k)$. Then $\prod f_{1} \geqslant \prod f_{2}$.

(34) Let us consider finite sequences $f_{1}, f_{2}$ of elements of $\mathbb{R}$. Suppose len $f_{1}=$ len $f_{2}$ and for every element $k$ of $\mathbb{N}$ such that $k \in \operatorname{dom} f_{2}$ holds $f_{1}(k) \geqslant$ $f_{2}(k) \geqslant 0$. Then $\prod f_{1} \geqslant \prod f_{2}$.

PROOF: For every real number $r$ such that $r \in \operatorname{rng} f_{2}$ holds $r \geqslant 0$. For every real number $r$ such that $r \in \operatorname{rng} f_{1}$ holds $r \geqslant 0$.

(35) Let us consider a positive real number $a$, and a non-negative yielding finite sequence $f$ of elements of $\mathbb{R}$. Suppose for every element $k$ of $\mathbb{N}$ such that $k \in \operatorname{dom} f$ holds $f(k) \leqslant a$. Then $\prod f \leqslant a^{\text {len } f}$. The theorem is a consequence of (20) and (33). 


\section{Basic Operations on Short Finsequences}

Let $a$ be a complex number. Let us note that $(-\langle-a\rangle)(1)$ reduces to $a$ and $\left(\left\langle a^{-1}\right\rangle^{-1}\right)(1)$ reduces to $a$.

Let us consider complex numbers $a, b$. Now we state the propositions:

(36) $\langle a\rangle+\langle b\rangle=\langle a+b\rangle$.

(37) $\langle a\rangle-\langle b\rangle=\langle a-b\rangle$. The theorem is a consequence of (36).

(38) $\langle a\rangle \cdot\langle b\rangle=\langle a \cdot b\rangle$.

(39) $\langle a\rangle /\langle b\rangle=\left\langle a \cdot\left(b^{-1}\right)\right\rangle$. The theorem is a consequence of (38).

Let $n$ be a natural number, $f$ be an $n$-element finite sequence, and $a$ be a complex number. One can verify that $\left(f^{\wedge}\langle a\rangle\right)(n+1)$ reduces to $a$ and $\left(f^{\wedge}\langle a\rangle\right)\lceil n$ reduces to $f$.

Let $a, b, c, d$ be complex numbers. Let us observe that $\langle a, b, c, d\rangle$ is complexvalued.

Let $a, b$ be complex numbers. Let us observe that $(-\langle-a, b\rangle)(1)$ reduces to $a$ and $(-\langle a,-b\rangle)(2)$ reduces to $b$ and $\left(\left\langle a^{-1}, b\right\rangle^{-1}\right)(1)$ reduces to $a$ and $(\langle a$, $\left.\left.b^{-1}\right\rangle^{-1}\right)(2)$ reduces to $b$.

Let $a, b, c$ be complex numbers. Note that $\langle a, b, c\rangle(1)$ reduces to $a$ and $\langle a, b$, $c\rangle(2)$ reduces to $b$ and $(-\langle-a, b, c\rangle)(1)$ reduces to $a$ and $(-\langle a,-b, c\rangle)(2)$ reduces to $b$ and $(-\langle a, b,-c\rangle)(3)$ reduces to $c$ and $\left(\left\langle a^{-1}, b, c\right\rangle^{-1}\right)(1)$ reduces to $a$ and $(\langle a$, $\left.\left.b^{-1}, c\right\rangle^{-1}\right)(2)$ reduces to $b$ and $\left(\left\langle a, b, c^{-1}\right\rangle^{-1}\right)(3)$ reduces to $c$.

Now we state the propositions:

(40) Let us consider complex numbers $a, b$, a natural number $n$, and $n$ element, complex-valued finite sequences $f, g$. Then $f^{\frown}\langle a\rangle+g^{\frown}\langle b\rangle=$ $(f+g)^{\frown}\langle a+b\rangle$.

Proof: Reconsider $f_{3}=f^{\frown}\langle a\rangle$ as an $(n+1)$-element finite sequence of elements of $\mathbb{C}$. Reconsider $g_{1}=g \frown\langle b\rangle$ as an $(n+1)$-element finite sequence of elements of $\mathbb{C}$. For every object $k$ such that $k \in \operatorname{dom}\left(f_{3}+g_{1}\right)$ holds $\left(f_{3}+g_{1}\right)(k)=((f+g) \frown\langle a+b\rangle)(k)$.

(41) Let us consider complex numbers $a, b, x, y$. Then $\langle a, b\rangle+\langle x, y\rangle=\langle a+x$, $b+y\rangle$. The theorem is a consequence of (40) and (36).

(42) Let us consider complex numbers $a, b, c, x, y, z$. Then $\langle a, b, c\rangle+\langle x, y$, $z\rangle=\langle a+x, b+y, c+z\rangle$. The theorem is a consequence of (40) and (41).

(43) Let us consider complex numbers $a, b, c, d, x, y, z, v$. Then $\langle a, b, c$, $d\rangle+\langle x, y, z, v\rangle=\langle a+x, b+y, c+z, d+v\rangle$. The theorem is a consequence of (40) and (42).

(44) Let us consider complex numbers $a, b$, a natural number $n$, and $n$ element, complex-valued finite sequences $f, g$. Then $\left(f^{\frown}\langle a\rangle\right) \cdot\left(g^{\frown}\langle b\rangle\right)=$ $(f \cdot g) \frown\langle a \cdot b\rangle$. 
Proof: Reconsider $f_{3}=f^{\frown\langle a\rangle}$ as an $(n+1)$-element finite sequence

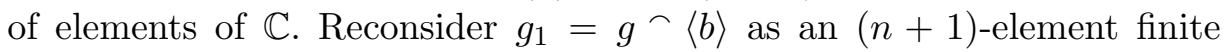
sequence of elements of $\mathbb{C}$. For every object $k$ such that $k \in \operatorname{dom}\left(f_{3} \cdot g_{1}\right)$ holds $\left(f_{3} \cdot g_{1}\right)(k)=((f \cdot g) \frown\langle a \cdot b\rangle)(k)$.

(45) Let us consider complex numbers $a, b, x, y$. Then $\langle a, b\rangle \cdot\langle x, y\rangle=\langle a \cdot x$, $b \cdot y\rangle$. The theorem is a consequence of (44) and (38).

(46) Let us consider complex numbers $a, b, c, x, y, z$. Then $\langle a, b, c\rangle \cdot\langle x, y$, $z\rangle=\langle a \cdot x, b \cdot y, c \cdot z\rangle$. The theorem is a consequence of (44) and (45).

(47) Let us consider complex numbers $a, b, c, d, x, y, z, v$. Then $\langle a, b, c, d\rangle \cdot\langle x$, $y, z, v\rangle=\langle a \cdot x, b \cdot y, c \cdot z, d \cdot v\rangle$. The theorem is a consequence of (44) and $(46)$.

(48) Let us consider a complex number $a$, a non zero natural number $n$, and an $n$-element, complex-valued finite sequence $f$. Then $\langle a\rangle+f=\langle a+f(1)\rangle$.

(49) Let us consider complex numbers $a, b$, a non trivial natural number $n$, and an $n$-element, complex-valued finite sequence $f$. Then $\langle a, b\rangle+f=$ $\langle a+f(1), b+f(2)\rangle$.

(50) Let us consider a complex number $a$, a non zero natural number $n$, and an $n$-element, complex-valued finite sequence $f$. Then $\langle a\rangle \cdot f=\langle a \cdot f(1)\rangle$.

(51) Let us consider complex numbers $a, b$, a non trivial natural number $n$, and an $n$-element, complex-valued finite sequence $f$. Then $\langle a, b\rangle \cdot f=$ $\langle a \cdot f(1), b \cdot f(2)\rangle$.

\section{ACKnowledgement: Ad Maiorem Dei Gloriam}

\section{REFERENCES}

[1] Grzegorz Bancerek and Krzysztof Hryniewiecki. Segments of natural numbers and finite sequences Formalized Mathematics, 1(1):107-114, 1990.

[2] Grzegorz Bancerek, Czesław Byliński, Adam Grabowski, Artur Korniłowicz, Roman Matuszewski, Adam Naumowicz, Karol Pąk, and Josef Urban. Mizar: State-of-the-art and beyond. In Manfred Kerber, Jacques Carette, Cezary Kaliszyk, Florian Rabe, and Volker Sorge, editors, Intelligent Computer Mathematics, volume 9150 of Lecture Notes in Computer Science, pages 261-279. Springer International Publishing, 2015. ISBN 978-3319-20614-1. doi 10.1007/978-3-319-20615-8_17.

[3] Grzegorz Bancerek, Czesław Byliński, Adam Grabowski, Artur Korniłowicz, Roman Matuszewski, Adam Naumowicz, and Karol Pak. The role of the Mizar Mathematical Library for interactive proof development in Mizar. Journal of Automated Reasoning, 61(1):9-32, 2018. do1 $10.1007 /$ s10817-017-9440-6

[4] Alexander Elizarov, Alexander Kirillovich, Evgeny Lipachev, and Olga Nevzorova. Digital ecosystem OntoMath: Mathematical knowledge analytics and management. In Leonid Kalinichenko, Sergei O. Kuznetsov, and Yannis Manolopoulos, editors, Data Analytics and Management in Data Intensive Domains, pages 33-46. Springer International Publishing, 2017.

[5] Adam Naumowicz and Artur Korniłowicz. A brief overview of Mizar. In International Conference on Theorem Proving in Higher Order Logics, pages 67-72. Springer, 2009. doi: $10.1007 / 978-3-642-03359-9 \_5$ 
[6] Piotr Rudnicki and Andrzej Trybulec. On the integrity of a repository of formalized mathematics. Mathematical Knowledge Management, 2003. doi $10.1007 / 3-540-36469-2 \_13$.

Accepted September 29, 2018 